\title{
A new methodology for studying neutron absorber materials: first results with boron carbide.
}

\author{
L. Desgranges ${ }^{1}$, J.M. Escleine ${ }^{1}$, Ph. Bienvenu ${ }^{1}$, I. Roure ${ }^{1}$, D. Gosset ${ }^{2}$, R. Boffy ${ }^{3}$, U. Köster ${ }^{3}$ \\ 1 CEA, DEN, DEC F-13108 Saint Paul lez Durance Cedex, France \\ 2 CEA, DEN, DMN, Université Paris-Saclay, F-91191 Gif-sur-Yvette Cedex, France \\ 3 Institut Laue-Langevin, 71 avenue des Martyrs, B.P. 156, 38042 Grenoble cedex, France
}

\begin{abstract}
A$ new methodology is presented for the study of transmutation damage in $\mathrm{B}_{4} \mathrm{C}$ boron carbide. The $\mathrm{B}_{4} \mathrm{C}$ samples are set in a specially designed sample holder, and irradiated in a neutron thermal flux, at the ILL reactor in our case. After the description of the sample holder and the irradiation conditions, the first post-irradiation examinations by Secondary Ion Mass Spectroscopy (SIMS), X-Ray Diffraction (XRD) and Secondary Electron Microscopy (SEM) are detailed. The first results obtained on a $\mathrm{B}_{4} \mathrm{C}$ disk with natural ${ }^{10} \mathrm{~B}$ composition prove the efficiency of the methodology.
\end{abstract}

\section{Introduction}

In the nuclear field, there is an increasing demand for more physically based modeling of material properties in order not only to have a limited number of validation irradiations in actual reactor conditions, but also to make more reliable predictions outside of the validation domain. This trend largely applies to nuclear fuel with numerous papers going from first principles calculations to modeling of the fuel microstructure [1]. It is also relevant for neutron absorber materials for which less work has been done. Boron carbide is one of the most used neutron absorbers and its phenomenology under irradiation has been for long documented $[2,3]$. In actual reactor conditions it is submitted to both atomic collision with fast neutrons and transmutation reactions due to neutron capture by the ${ }^{10} \mathrm{~B}$ isotope. Neutron atomic collisions produce collision cascades that result in point or extended defects. ${ }^{10} \mathrm{~B}$ transmutation reactions (up to $10^{22} \mathrm{~cm}^{-3}$, i.e. about 10 percent of total boron in actual fast neutron reactor conditions) produces $\mathrm{He}$ and $\mathrm{Li}$ recoils that induce defect formation during their stopping and chemical modification of the pristine boron carbide. Helium clusters in over-pressurized small bubbles could lead to inter- and intragranular cracking [4]. The fate of lithium is less clear since this element is not visible using classical methods such as Electron Probe Micro Analysis (EMPA) or X-Ray Photoelectron Spectroscopy (XPS).

A physically based modeling requires the identification of each physical mechanism that is relevant for the description of the material. In the case of boron carbide under irradiation, the effects of atomic collisions with fast neutrons and transmutation reactions cannot be easily separated from the available experimental results obtained by irradiations in nuclear reactors or ion implantation experiments. During a technological irradiation, the material is submitted to a wide neutron energy spectrum leading to a complex weighting of the two interactions, absorption and ballistic damage; thus ballistic and transmutation effects are intricately linked. With ion implantation, a suitable choice of the ion and its energy can reproduce one aspect of irradiation defects, but it fails to reproduce the 
transmutation phenomenon as a whole. Moreover each mechanism has to be characterized as a function of temperature. This results in the need of samples, irradiated at a constant temperature over a volume large enough to perform post-irradiation characterization. In technological irradiations, the temperature follows a complex history as a function of the reactor power and it also varies spatially as a function of the neutron flux. In implantation experiments the temperature may be known with a better accuracy, but the irradiated zone consists in a layer, usually less than $1 \mu \mathrm{m}$ thick, for which only few experimental methods, such as TEM, can provide valuable information, but which is not well suited for X-ray diffraction for example.

In order to overcome these difficulties, we developed a new methodology for studying neutron absorber materials in which only transmutation defects are produced within bulk samples at a nearly constant temperature. This methodology uses irradiation of $\mathrm{B}_{4} \mathrm{C}$ disks within a new sample holder in the high flux reactor at the Institut Laue Langevin (ILL). In a first part the sample holder is described, the irradiation and the first characterization results are then presented, and finally these results are discussed showing how the new methodology fulfilled our requirements.

\section{Sample holder}

In order to fit into the T4 beam tube, samples are settled in an aluminum shuttle. A new sample holder was designed to achieve the irradiation objectives presented above and also to fit into this shuttle. A schematic description of this sample holder is given in Figure 1.

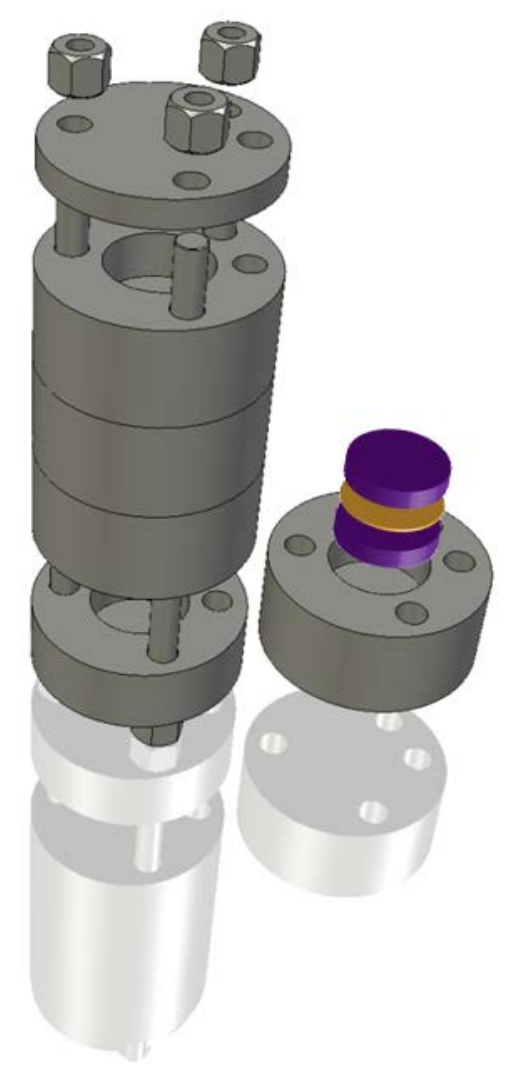


Figure 1: Schematic view of the $\mathrm{B}_{4} \mathrm{C}$ samples (in orange) sandwiched between two SiC disks (in purple) settled in their sample holder (in gray).

The $\mathrm{B}_{4} \mathrm{C}$ samples consist of $0.3 \mathrm{~mm}$ thick, $10 \mathrm{~mm}$ diameter disks that have been prepared from a high density $(>98 \%$ of theoretical density $=2.52)$, small grain size $(5-10 \mu \mathrm{m})$ pellet and which two faces have been mirror polished. Two $\beta$-SiC disks, $2 \mathrm{~mm}$ thick and $10 \mathrm{~mm}$ diameter, are put on each face of each $\mathrm{B}_{4} \mathrm{C}$ sample. $\beta$-SiC (theoretical density $=3.51$ ) disks are prepared from a CVD-made ceramic pellet and have the face in contact with the $\mathrm{B}_{4} \mathrm{C}$ sample that is mirror polished. Each $\mathrm{SiC}-\mathrm{B}_{4} \mathrm{C}-\mathrm{SiC}$ sandwich is installed inside a hole fitted in an aluminum disc. Five aluminum discs were fabricated and stacked one over the other in order to build the sample holder. The ${ }^{10} \mathrm{~B}$ enrichment of the samples was natural (19.9\%) for the three $\mathrm{B}_{4} \mathrm{C}$ bottom disks, $48 \%$ for the forth disc and $90 \%$ for the top one. The aluminum discs are kept together by three screws. An additional hole goes through all discs in order to let air flow out when the sample holder is put in the capsule. Small zirconium foils were inserted in this hole at different heights in order to determine the neutron flux using postirradiation gamma spectrometry. Figure 2 presents the sample holder when it was built up (Figure 2a) and when it was put into the capsule (Figure 2b).

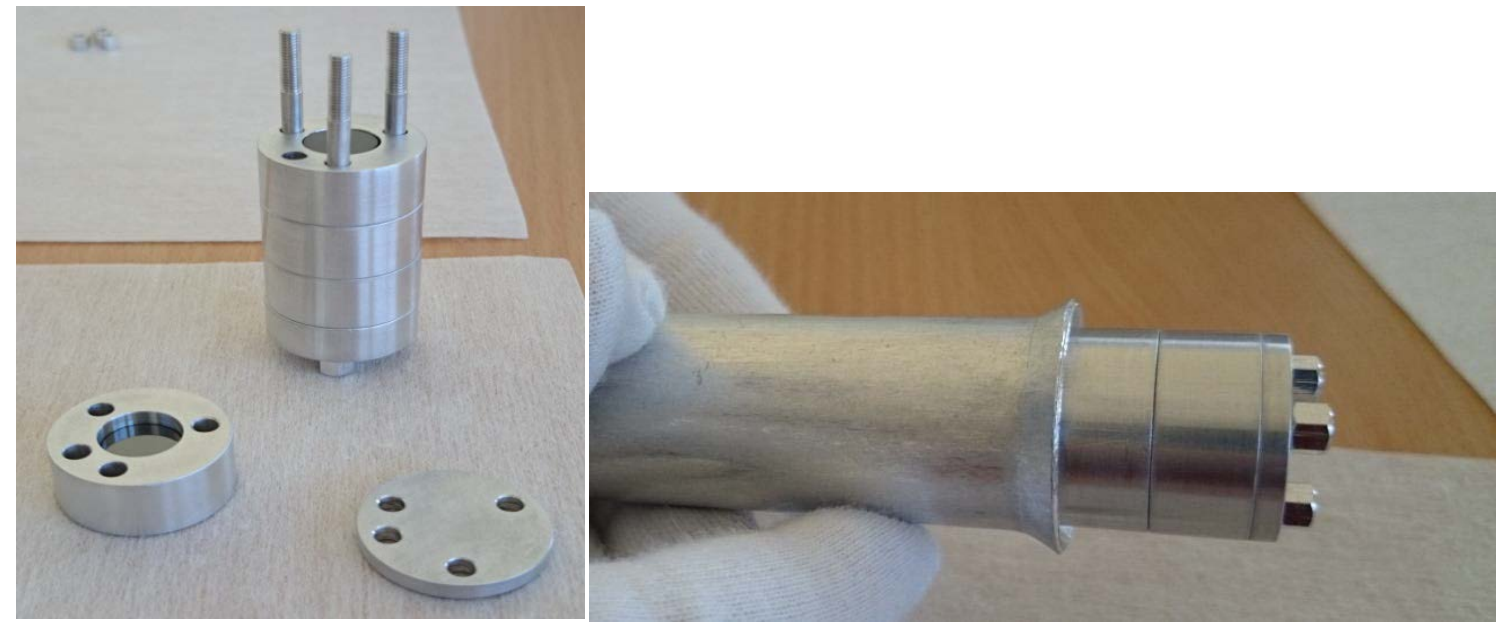

Figure 2: Sample holder fabrication and installation in the aluminum capsule.

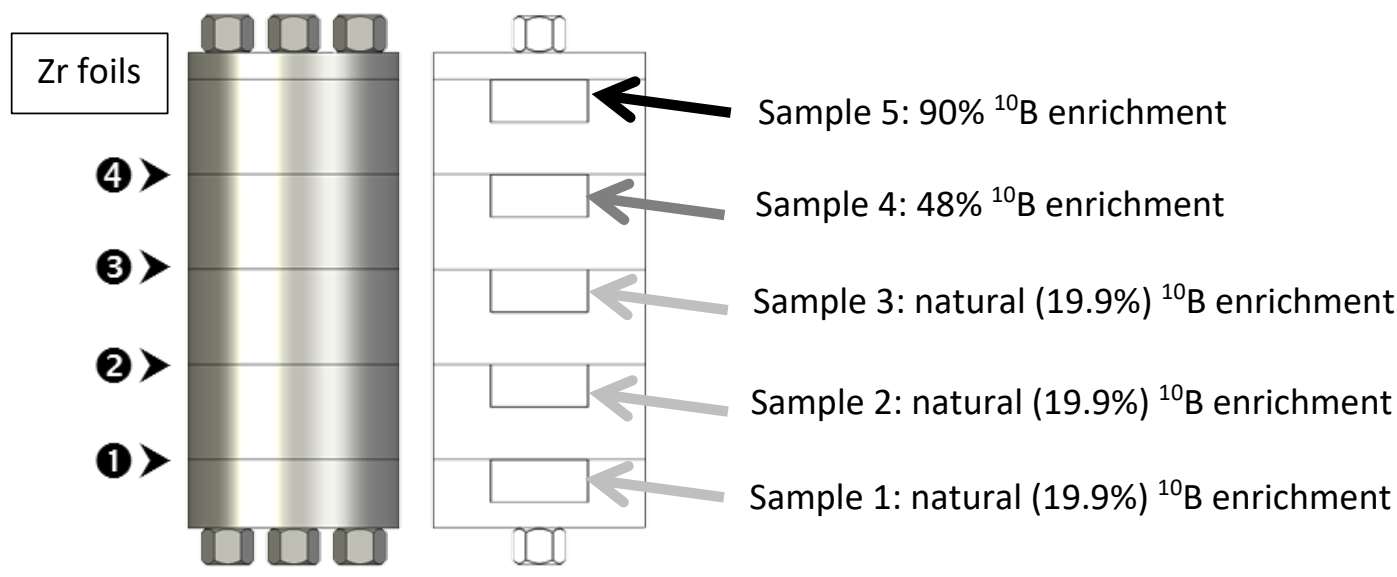


Figure 3: position of the zirconium flux monitor foils (white numbers on black dots) and ${ }^{10} \mathrm{~B}$ enrichment of the samples

This design also allows the safe irradiation of the sample holder within the capsule in the T4 tube. The high thermal conductivity of SiC and the quality of the contact between the mirror polished surfaces of $\mathrm{B}_{4} \mathrm{C}$ and $\mathrm{SiC}$ allows the efficient removal of the heat produced by the transmutation reactions. Moreover the irradiation time was fixed to 5 days in order not only to produce a sufficient level of transmutations that will allow further characterization, but also to minimize the helium production and conservatively (i.e. assuming total helium release) keep the increase of pressure within the capsule to a value less than one atmosphere.

\section{Irradiation}

T4 irradiation tube is located within the reflecting light water vessel, very close to the heavy water tank, in the ILL reactor. This location leads to a neutron flux with a Maxwellian distribution for a temperature about $20^{\circ} \mathrm{C}$ that was calculated in [5].

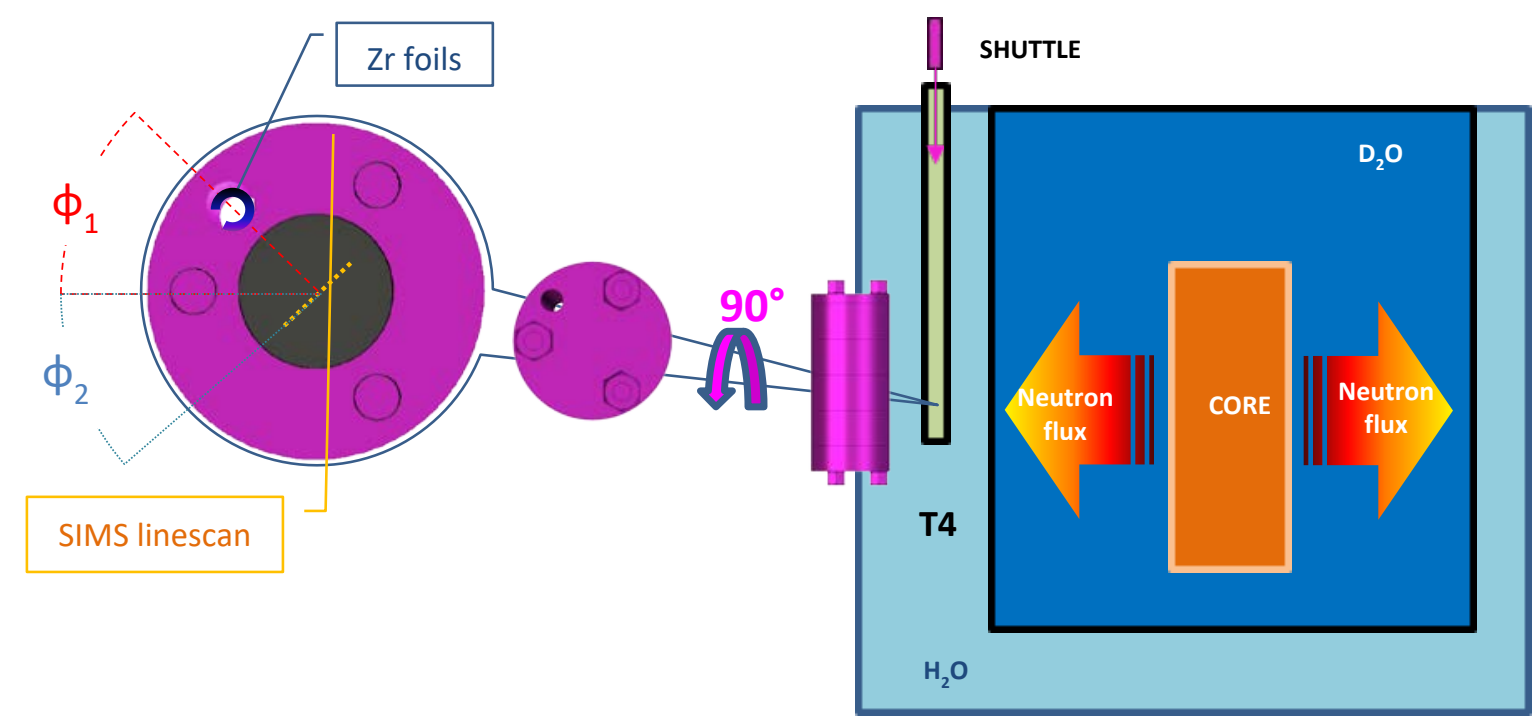

Figure 4: layout of T4 tube inside ILL reactor (left) and relative position of inside the sample holder relatively to the neutron flux.

The shuttle is set vertically in $\mathrm{T} 4$ tube so that the $\mathrm{B}_{4} \mathrm{C}$ disks lie parallel to the neutron flux coming from the core. Unfortunately the position of the sample holder inside T4 cannot be known. As a consequence, the relative position of the zirconium foils $(\phi 1)$ and the SIMS quantitative linescan $(\phi 2$ see later in the text) are not known relative the neutron flux. But this neutron flux has large local 
variation because of nuclear reaction with ${ }^{10} \mathrm{~B}$ that reduces the flux at the $\mathrm{B} 4 \mathrm{C}$ disk neighborhood. Not knowing $\phi 1$ and $\phi 2$ induces large inaccuracy in the neutron flux at the position of the zirconium foils and SIMS linescan respectively. Consequently we did not attempt to model the neutron flux theoretically, but we preferred relying on its experimental determination .

The penetration depth of $0.025 \mathrm{eV}$ thermal neutrons in natural $\mathrm{B}_{4} \mathrm{C}$ is about $130 \mu \mathrm{m}\left(\sigma \mathcal{N}=76 \mathrm{~cm}^{-1}\right)$. Thus the irradiation damage is more or less homogeneous in our $300 \mu \mathrm{m}$ thick $\mathrm{B}_{4} \mathrm{C}$ disks that have natural ${ }^{10} \mathrm{~B}$ enrichment, despite the local neutron flux changes discussed above.

The capsule containing the sample holder and the five SiC-B $\mathrm{B}_{4} \mathrm{C}-\mathrm{SiC}$ sandwiches was irradiated during five days in June 2015. Because of radiation level of the capsule after irradiation, it could be taken out of the hot cell only in September 2015. In a first step, the two ends of the capsule were cut and the small zirconium foils were taken out of the hole allowing air flow. After measuring them by gamma spectrometry, their accumulated dose could be calculated and the neutron flux during irradiation has been determined at different height inside the sample holder, Table 1 . The average measured flux is consistent with the nominal flux in T4 [6].

\begin{tabular}{|l|l|l|l|l|l|}
\hline Foil number & $\mathbf{1}$ & $\mathbf{2}$ & $\mathbf{3}$ & $\mathbf{4}$ & average \\
\hline Foil mass $(\mathrm{mg})$ & 1.59 & 1.63 & 1.71 & 1.35 & \\
\hline $\begin{array}{l}\text { Calculated flux } \\
\text { in } 10^{13} \mathrm{n} / \mathrm{cm}^{2} / \mathrm{s}\end{array}$ & 1.8 & 1.73 & 1.39 & 2.18 & $1.7+/-.3$ \\
\hline
\end{tabular}

Table 1 : calculated neutron flux at the different zirconium foil positions

In a second step, the capsule was sent to CEA-Caradache where it was dismantled and the $\mathrm{SiC}-\mathrm{B}_{4} \mathrm{C}-\mathrm{SiC}$ sandwiches could be removed. The radioactivity of the $\mathrm{SiC}$ and $\mathrm{B}_{4} \mathrm{C}$ disks was measured. For each sample, the dose rate was less than $10^{-4} \mathrm{mSv} / \mathrm{h}$ at $5 \mathrm{~cm}$ distance and the labile contamination was less than $4 \mathrm{~Bq} \cdot \mathrm{cm}^{-2}$ (for alpha and beta activity respectively). This low radioactivity level (i.e. exempt classification) allows the samples to be handled in regular facilities without significant limitations due to radioactivity.

\section{First characterizations}

The sandwich with natural boron composition located in the middle of the capsule was chosen for further analysis. The two $\mathrm{SiC}$ disks were separated from the $\mathrm{B}_{4} \mathrm{C}$ sample. The $\mathrm{B}_{4} \mathrm{C}$ sample and one $\mathrm{SiC}$ disk were stuck on standard SEM $12.7 \mathrm{~mm}(1 / 2 ")$ pin stubs for further analyses by SIMS and XRD. Preliminary SEM analysis evidenced that a surface film, a few hundreds of nanometer thick, has been removed out from some parts of the $\mathrm{B}_{4} \mathrm{C}$ disk. This film is likely to be fabrication pollution, a small fraction of graphite present in the material (free carbon, resulting from the elaboration process). In the following we will focus mainly on the behavior of bulk $B_{4} C$.

\section{$\underline{B}_{4}$ C sample}


The $\mathrm{B}_{4} \mathrm{C}$ disc was characterized first by SIMS to evidence the change in isotopic composition induced by irradiation and second by SEM and XRD to evidence the defects created by ${ }^{10} \mathrm{~B}$ transmutation.

SIMS analyses were performed using a positive oxygen primary beam of $4.5 \mathrm{keV}$ energy and measuring positive secondary ions. The SIMS depth profiles were performed for ${ }^{10} \mathrm{~B},{ }^{11} \mathrm{~B}$ and ${ }^{12} \mathrm{C}$ that existed before irradiation and for ${ }^{7} \mathrm{Li}$ that was created during irradiation. The SIMS signal is not proportional to the isotope concentration; it also depends on the ionization yield. Thus, these depth profiles should not be compared quantitatively the one to the other.

Isotopic depth profiles were performed at different points of the $\mathrm{B}_{4} \mathrm{C}$ surface. They all evidenced two regions with different detection yields for $B, C$ and $L i$ ions: one region lies at the near surface while the other is in the bulk (figure 4). The SIMS crater depth was measured to be around one $\mu \mathrm{m}$ deep. This confirms that the first region is a few hundred nanometers thick and that it is associated to the graphite surface film presented before.

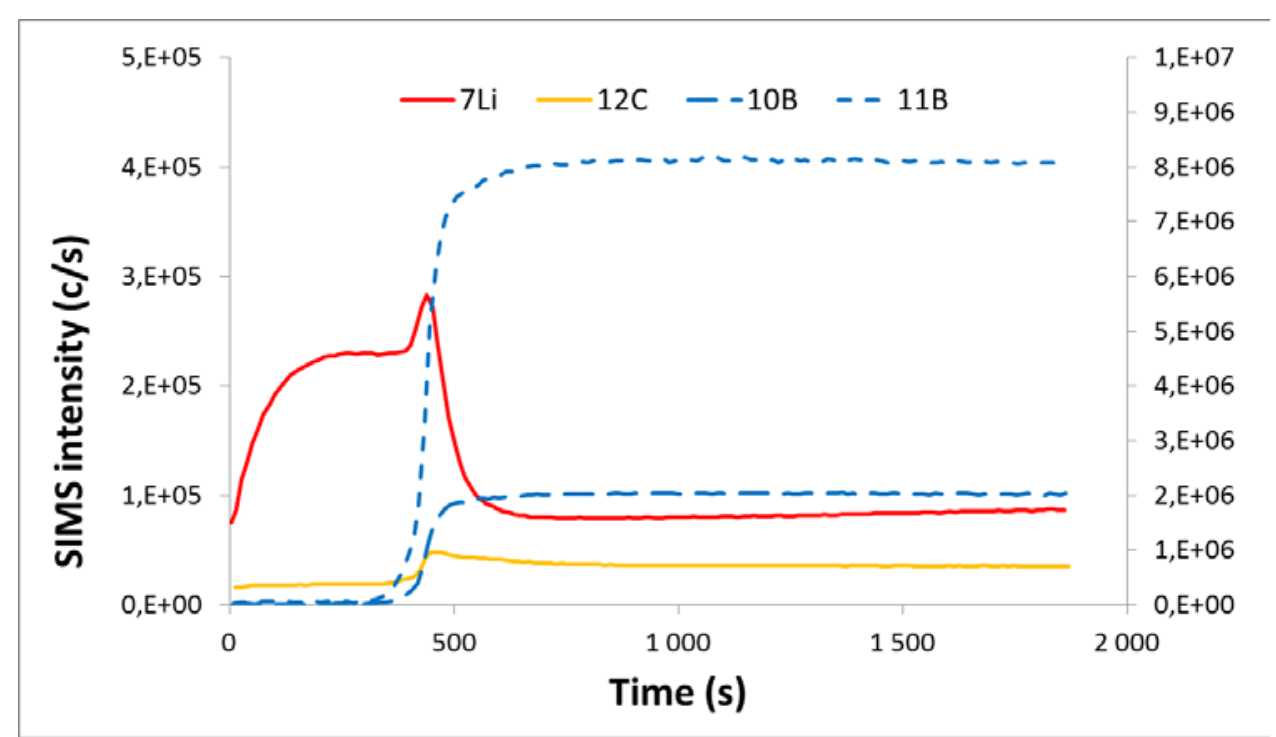

Figure 4. Depth profiles of ${ }^{12} \mathrm{C},{ }^{10} \mathrm{~B},{ }^{11} \mathrm{~B}$ and ${ }^{7} \mathrm{Li}$ obtained by SIMS in the $\mathrm{B}_{4} \mathrm{C}$ sample. The scale for the dashed curves is on the left side, the scale for the plain curves on the right side.

For sputtering times longer than $700 \mathrm{~s}$, that correspond to the bulk sample, B and C profiles are flat consistently with the neutron absorption length in $B_{4} C$ with natural ${ }^{10} B$ composition $(130 \mu \mathrm{m})$ that induces no significant change in transmutation range over a $1 \mu \mathrm{m}$ depth. On the contrary, the lithium profile increases on that timeframe. This implies that the lithium created by transmutation reactions moved out of the $\mathrm{B}_{4} \mathrm{C}$ bulk. ${ }^{7} \mathrm{Li}$ is produced as a recoil atom created during ${ }^{10} \mathrm{~B}$ transmutation; its recoil energy enables it to move a few $\mu \mathrm{m}$ away from its creation place. Recoil implantation in SiC (see later) is then consistent with a depletion of $\mathrm{Li}$ in $\mathrm{B}_{4} \mathrm{C}$. But the peak at circa $400 \mathrm{~s}$ in the lithium profile on Figure 4 is likely a sign for Li accumulation, suggesting that Li desorption out of $\mathrm{B}_{4} \mathrm{C}$ should also be considered [7]. This peak might also be correlated to small particles that were evidenced by $\mathrm{SEM}$ on the $\mathrm{B}_{4} \mathrm{C}$ surface. These particles are spheroid shaped; they are deposited on the surface, either isolated or forming clusters (figure 5). The dismantling procedure took care of avoiding contamination deposition on the samples, and these spheroids should have been created during the 
irradiation at ILL. A better characterization of these spheroids is needed for a better understanding of the Li behavior.

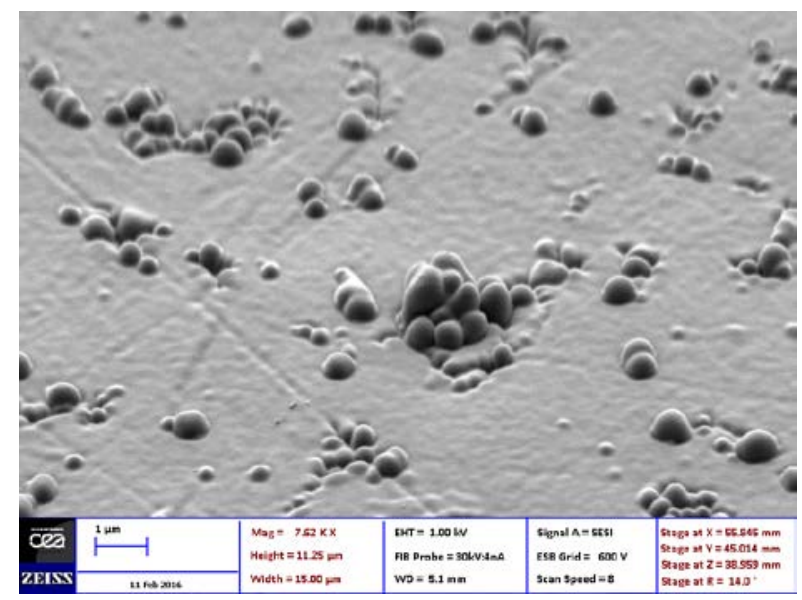

Figure 5. Spheroid particles at the surface of the $\mathrm{B}_{4} \mathrm{C}$ disc.

A quantitative determination of the ${ }^{10} \mathrm{~B} /{ }^{11} \mathrm{~B}$ ratio was also performed. For that purpose, the surface film was previously sputtered to allow the measurement in the bulk where the ${ }^{10} \mathrm{~B}$ and ${ }^{11} \mathrm{~B}$ concentrations are constant (figure 4). Ten equidistant measuring points were chosen in a line on disk surface.
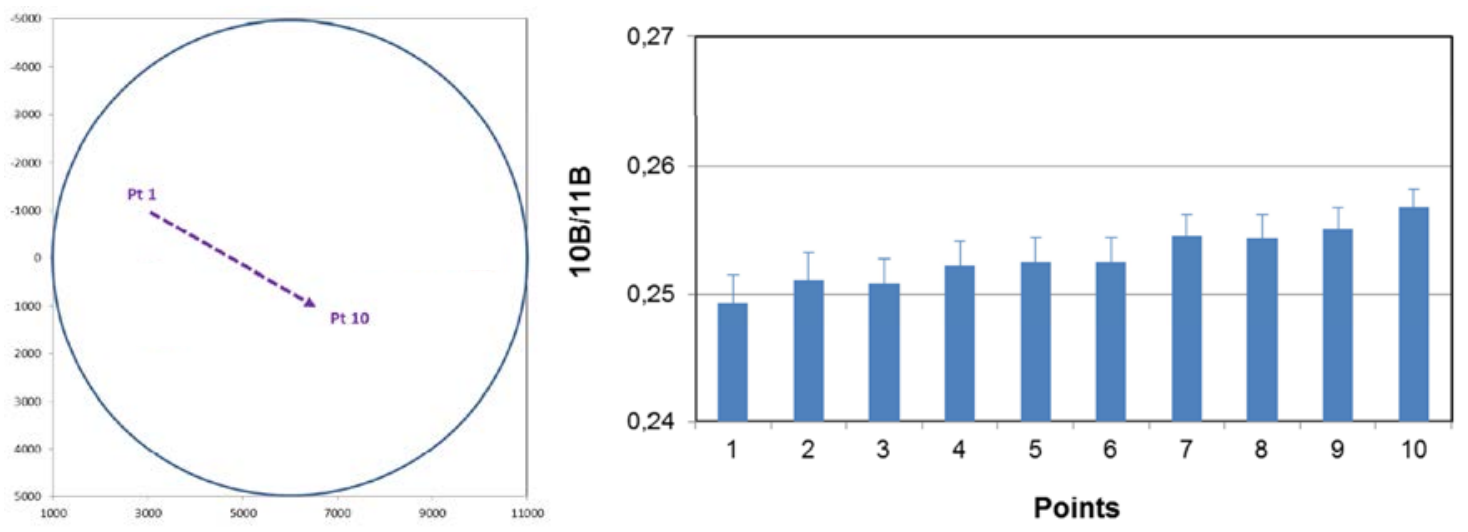

Figure $6:{ }^{10} \mathrm{~B} /{ }^{11} \mathrm{~B}$ measured isotopic ratio along the radius of the $\mathrm{B}_{4} \mathrm{C}$ disc, after removal of the surface film.

The measured ${ }^{10} \mathrm{~B} /{ }^{11} \mathrm{~B}$ ratio was corrected for the experimental isotopic drift, using a $\mathrm{B}_{4} \mathrm{C}$ reference with a known ${ }^{11} \mathrm{~B}$ composition. The corrected ${ }^{10} \mathrm{~B} /{ }^{11} \mathrm{~B}$ ratio is 0.2529 with a standard deviation of the order of $1 \%$. This value is significantly lower and more dispersed than the 0.2645 with $0.3 \%$ standard deviation measured on unirradiated $\mathrm{B}_{4} \mathrm{C}$ coming from the same fabrication batch, which confirms that significant transmutation occurred. Moreover the higher standard deviation for irradiated $\mathrm{B}_{4} \mathrm{C}$ can be attributed to an evolution of the ${ }^{10} \mathrm{~B} /{ }^{11} \mathrm{~B}$ ratio the decreases along the disk radius (Figure 6), consistently with a continuous decrease of the neutron flux in B4C disk in the direction escaping the reactor core. 
$\mathrm{X}$-ray diffraction was performed using $\mathrm{Cu} \mathrm{K} \alpha_{1}$ radiation on an asymmetric goniometer. The primary beam (width $100 \mu \mathrm{m}$ ) hits the sample surface at a fixed angle of $2^{\circ}$. This small angle diffraction set-up was chosen in order to limit the penetration of $X$-rays inside the $B_{4} C$ sample because $B_{4} C$ is a low $Z$ material [8]. The estimated penetration depth of $X$-rays in our low angle diffraction set-up (incidence angle $\left.\mathrm{i}=2^{\circ}\right)$ is about $60 \mu \mathrm{m}\left(\mu / \sin (\mathrm{i})=170 \mathrm{~cm}^{-1}\right)$. As a result, the material analyzed by XRD can be considered as homogeneous, apart the pollution surface layer already discussed.

The XRD diffraction diagram of the irradiated $\mathrm{B}_{4} \mathrm{C}$ is compared to the reference on figure 7. Peak shifts and changes in peak intensity can easily be observed showing that the crystalline structure of $\mathrm{B}_{4} \mathrm{C}$ was damaged by stopping $\mathrm{He}$ and Li produced by transmutation. A small fraction of graphite present in the material (free carbon, resulting from the elaboration process) shows a significant decrease after irradiation, due to disorder or partial amorphisation. A further interpretation of the XRD data using Rietveld analysis is in progress.

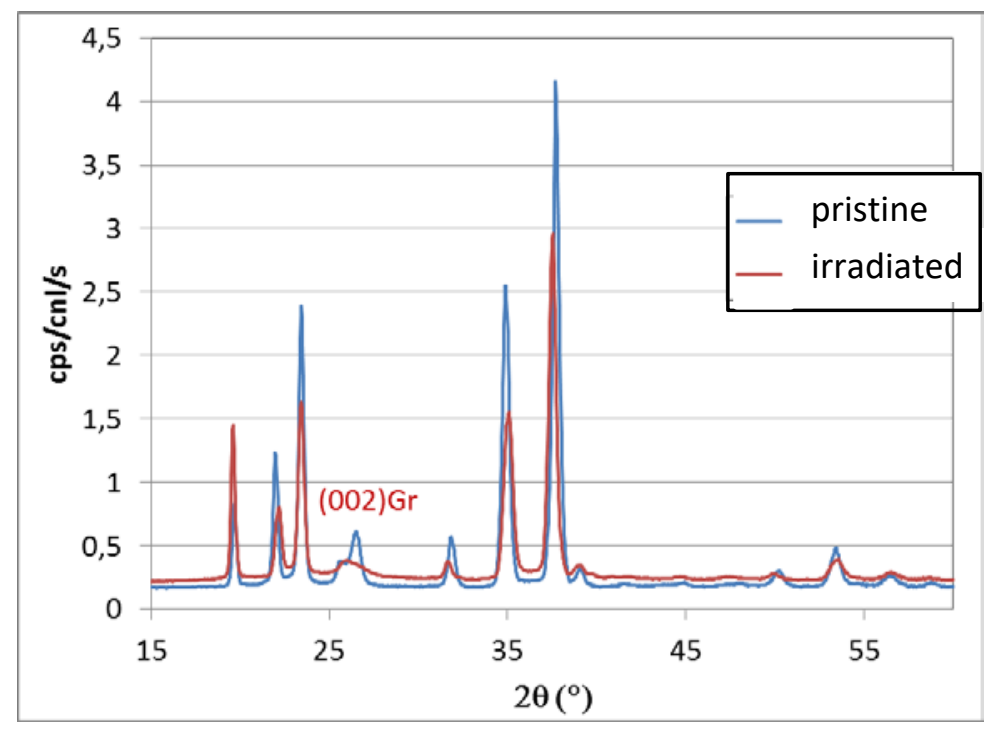

Figure 7. X-ray diffraction diagrams before (blue) and after (red) irradiation. The (002)Gr line corresponds to extra-graphite present in the material at about $2000 \mathrm{ppm}$.

\section{$\underline{\text { SiC sample }}$}

In a similar manner to $\mathrm{B}_{4} \mathrm{C}$, the $\mathrm{SiC}$ disk face that was in contact with the $\mathrm{B}_{4} \mathrm{C}$ sample was also characterized by SIMS and XRD.

The SIMS depth profile also evidenced a near surface altered layer (figure 8). This should be the same layer previously observed on $\mathrm{B}_{4} \mathrm{C}$, partially stuck to the $\mathrm{SiC}$ disk. The ${ }^{7} \mathrm{Li}$ profile evidenced that $\mathrm{Li}$ was implanted in $\mathrm{SiC}$ with a decreasing intensity towards the $\mathrm{SiC}$ bulk. This decrease is consistent with the implantation of Li recoil atoms because the implantation depth of lithium can be estimated around a few micrometers and the SIMS crater depth was measured by profilometry around $0,8 \mu \mathrm{m}$. 


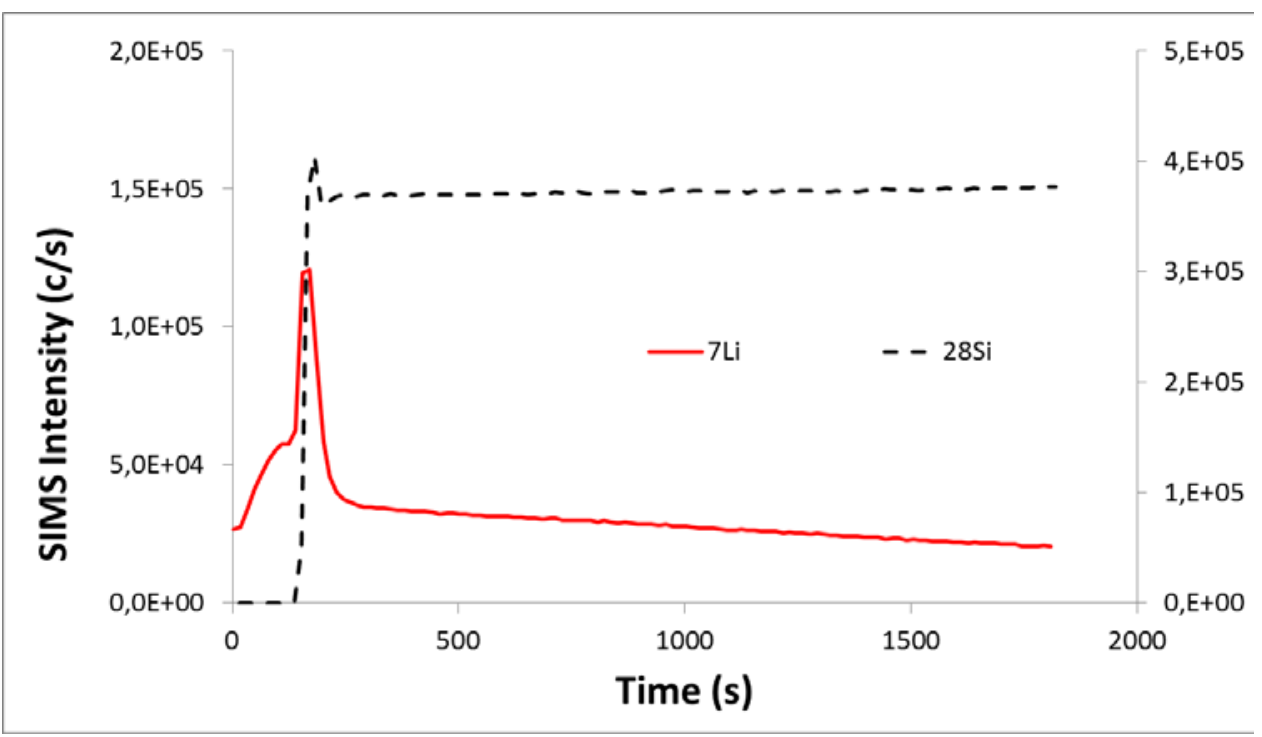

Figure 8: SIMS profiles from the SiC surface in contact with the $\mathrm{B}_{4} \mathrm{C}$ disc. The scale of the dashed curve is on the left side, the scale of the plain curve on the right side.

Figure 9 shows XRD pattern of reference and irradiated SiC. The irradiated SiC was measured on the face that was in contact with $\mathrm{B}_{4} \mathrm{C}$ during irradiation. This figure evidences damage formation in the $\mathrm{SiC}$ that appears through peak broadening, intensity change and shifting.

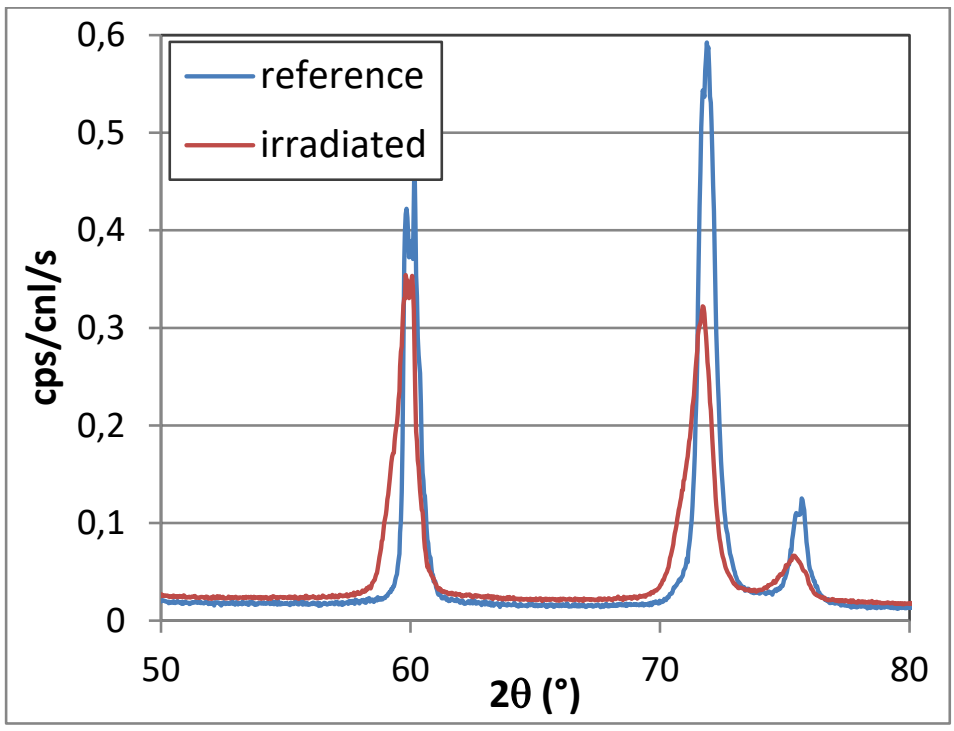

Figure 9. XRD diagrams of the surface of the $\mathrm{SiC}$ disc in contact with the $\mathrm{B}_{4} \mathrm{C}$ disc.

\section{Discussion}

In this irradiation we aimed at getting $\mathrm{B}_{4} \mathrm{C}$ samples in which only transmutation defects are produced within bulk samples at a given temperature. The results presented here demonstrated that this objective is fulfilled. 
First the very pure thermal neutron flux in T4 induces no damage due to neutron collisions with atoms in the $\mathrm{B}_{4} \mathrm{C}$ samples because the energy transferred by neutron collisions to a $\mathrm{B}$ or $\mathrm{C}$ atom is less than its displacement threshold. Moreover the neutron absorption cross-section for the reaction

$$
{ }^{10} \mathrm{~B}+\mathrm{n}-->{ }^{7} \mathrm{Li}+{ }^{4} \mathrm{He}
$$

is very high (3840 barns). Therefore only transmutation induced defects are created: chemical modification, ions slowing down (mainly electronic interactions with the matrix) and stopping (partly ballistic damage).

The transmutation dose can be determined using two different methods, the first one using neutron flux evaluation, the other one using SIMS results. First, the neutron flux during irradiation was evaluated around $1.8 \cdot 10^{13} \mathrm{n} \cdot \mathrm{cm}^{-2} \cdot \mathrm{s}^{-1}$ using the zirconium foils. Integrated over the duration of the irradiation, this leads to about $6 \cdot 10^{20}$ neutron captures $/ \mathrm{cm}^{3}$, which corresponds to a $3.4 \%$ transmutation yield of ${ }^{10} \mathrm{~B}$ and $0.6 \%$ burn-up of total boron. Second, the ${ }^{10} \mathrm{~B} /{ }^{11} \mathrm{~B}$ ratio measured by SIMS before and after irradiation can also be used to evaluate the fraction of ${ }^{10} \mathrm{~B}$ that underwent transmutation; a concentration of about $9 \cdot 10^{20}$ neutron captures $/ \mathrm{cm}^{3}$ was deduced, which corresponds to a $4.4 \%$ transmutation rate of ${ }^{10} \mathrm{~B}$. These two evaluations gave roughly consistent results regarding the experimental uncertainties. Preliminary tests in order to determine the $\mathrm{Li}$ concentration by SIMS are also consistent with these evaluations. Referring to literature, a transmutation dose of $10^{21}$ neutron captures $/ \mathrm{cm}^{3}$ corresponds to the dose at which irradiation effects have a noticeable effect on the material properties, such as structural damage or helium bubble formation at high temperature. Higher doses are available with the ${ }^{10} \mathrm{~B}$ enriched samples that were also irradiated.

The irradiation temperature has been evaluated before irradiation with conservative hypothesis to be lower than $600^{\circ} \mathrm{C}$. However this estimation is very crude, especially because of the local neutron flux changes discussed before. Therefore we plan to deduce the irradiation temperature from postexamination of $\mathrm{SiC}$ disks that were in contact with the $\mathrm{B}_{4} \mathrm{C}$ samples. $\mathrm{SiC}$ samples do not react with thermal neutrons but they can be damaged by transmutation recoils produced near the $\mathrm{B}_{4} \mathrm{C}$ surface. ${ }^{7} \mathrm{Li}$ and ${ }^{4} \mathrm{He}$ created by reaction (1) have recoil energies of $0.84 \mathrm{MeV}(94 \%), 1.01 \mathrm{MeV}(6 \%)$ for ${ }^{7} \mathrm{Li}$ and $1.47 \mathrm{MeV}(94 \%), 1.78 \mathrm{MeV}(6 \%)$ for ${ }^{4} \mathrm{He}$ respectively. When being produced close to the $\mathrm{B}_{4} \mathrm{C}$ surface, they can enter SiC. For example, the stopping length for $1 \mathrm{MeV}^{7} \mathrm{Li}$ in $\mathrm{SiC}$ is about $1.8 \mu \mathrm{m}$. Such an implantation depth for $\mathrm{Li}$ is consistent with the SIMS measured Li profile in SiC. X-ray diffraction was performed in the same conditions as for $\mathrm{B}_{4} \mathrm{C}$. This implies that the analyzed depth, here about $7 \mu \mathrm{m}$, is larger than the implantation range of $\mathrm{Li}$, and reveals some damage (figure 9). Now, it is known that some irradiation damage in SiC can be annealed when heated at their previous irradiation temperature [9]. So the $\mathrm{SiC}$ face, which is damaged by the transmutation recoil, can be heat treated to determine the annealing temperature of irradiation damage that corresponds to the irradiation temperature. This still has to be done.

\section{Conclusion}

We proposed a new methodology for the study of neutron absorber materials based on irradiation of $\mathrm{B}_{4} \mathrm{C}$ samples in a pure thermal neutron flux at the ILL reactor. The $\mathrm{B}_{4} \mathrm{C}$ samples obtained are 
homogeneously damaged in the bulk at a temperature that could be known in the near future. After some decay the samples are basically not radioactive, that makes them well suited for further studies aimed at characterizing transmutation damage. Some of the next objectives will be to analyze the structural damages in $\mathrm{B}_{4} \mathrm{C}$, the possible occurrence of helium clusters and determine at which temperature transmutation damage is annealed in $\mathrm{B}_{4} \mathrm{C}$ so as to identify which of these are relevant for fast breeder operating conditions. The characterization of the ${ }^{10} \mathrm{~B}$ enriched $\mathrm{B}_{4} \mathrm{C}$ disks that were also irradiated should provide some valuable information by comparison with the sample studied here.

In addition to scientific objectives, some technical improvements can be made to make easier the handling of irradiated samples. The gamma dose rate of the sample holder could be reduced by using $\mathrm{SiC}$ instead of aluminum for the fabrication of the sample holder. And the near surface layer observed after irradiation could be avoided using an improved polishing procedure for $\mathrm{B}_{4} \mathrm{C}$.

\section{References}

[1] Bertolus, Marjorie; Freyss, Michel; Dorado, Boris; et al., Linking atomic and mesoscopic scales for the modelling of the transport properties of uranium dioxide under irradiation, J. Nucl. Mater. 462 (2015) 475-495

[2] J.A. Basmajian, A.L. Pitner, D.E. Mahagin, H.C.F. Rippel and D.E. Baker; Irradiation effects in boron carbide pellets irradiated in a fast neutron spectra; Nucl. Tech. 16 (1972), 238-248

[3] D. Gosset, Absorber materials, in "Sodium Fast Reactor Design: Fuels, Neutronics, Thermal-Hydraulics, Structural Mechanics and Safety", Handbook of Nuclear Engineering, Dan Gabriel Cacuci (ed.), Springer (2010)

[4] G.W. Hollenberg, Effect of fast neutron irradiation on the structure of boron carbide, J. Am. Cer. Soc., 6011-12 (1977), 520-525

[5] A. Letourneau et al. Annals of Nuclear Energy 33 (2006) 377-384

[6 ] Boffy, R.; Peuget, S.; Schweins, R.; et al. "High thermal neutron flux effects on structural and macroscopic properties of alkali-borosilicate glasses used as neutron guide substrate" Nucl. Instum. Methods B 374 (2016) 14-19

[7] D. Simeone, X. Deschanels, B. Berthier, C. Tessier, Experimental evidence of lithium migration out of an irradiated boron carbide material, J. Nucl. Mater. 245 (1997) 27-33

[8] D. Gosset, D. Simeone, D, Quirion, Neutron irradiation damage of boron carbide: an X-ray diffraction evaluation, J. Phys. IV France Pr10 (2000) 55-63

[9] J.E Palentine « the development of silicon carbide as a routine irradiation temperature monitor, and its calibration in a thermal reactor » J. Nucl. Mater. 61 (1976) 243-253 